\title{
Multiple Period Resonances of Long Period Gratings in Photonic Crystal Fiber
}

\author{
Jovana S. Petrovic, Vladimir Mezentsev, Helen Dobb, David Webb, and Ian Bennion \\ Photonics Research Group, Aston Univeristy, Aston Triangle, Birmingham, B4 7ET, United Kingdom \\ petrovij@aston.ac.uk
}

\begin{abstract}
A comprehensive eigenmode analysis is performed of the guided modes supported by typical photonic crystal fiber. These modes exhibit unusual phase matching conditions requiring multiple grating periods for resonant coupling. All the signature features of the experimentally observed transmission spectra are explained by multiple-period resonances

(C) 2005 Optical Society of America

OCIS codes: ( ) word;
\end{abstract}

\section{Introduction}

Long period gratings (LPGs) consist of a large-scale (typically hundreds of microns) periodic axial perturbation in refractive index of the core or waveguiding geometry of an optical fibre. In recent years, LPGs have been increasingly used in a wide variety of sensing and telecommunications sensing applications. Periodic nature of LPGs allows for efficient resonant coupling between guiding modes, for example, between the fundamental and the cladding modes. A necessary condition of such a resonance is phase matching between these modes, when the intermodal beat period equals the grating period. The resulting effect of an LPG in transmission spectrum is usually a set of well defined attenuation bands corresponding to resonances between core mode and different cladding modes as shown in Fig. 1.

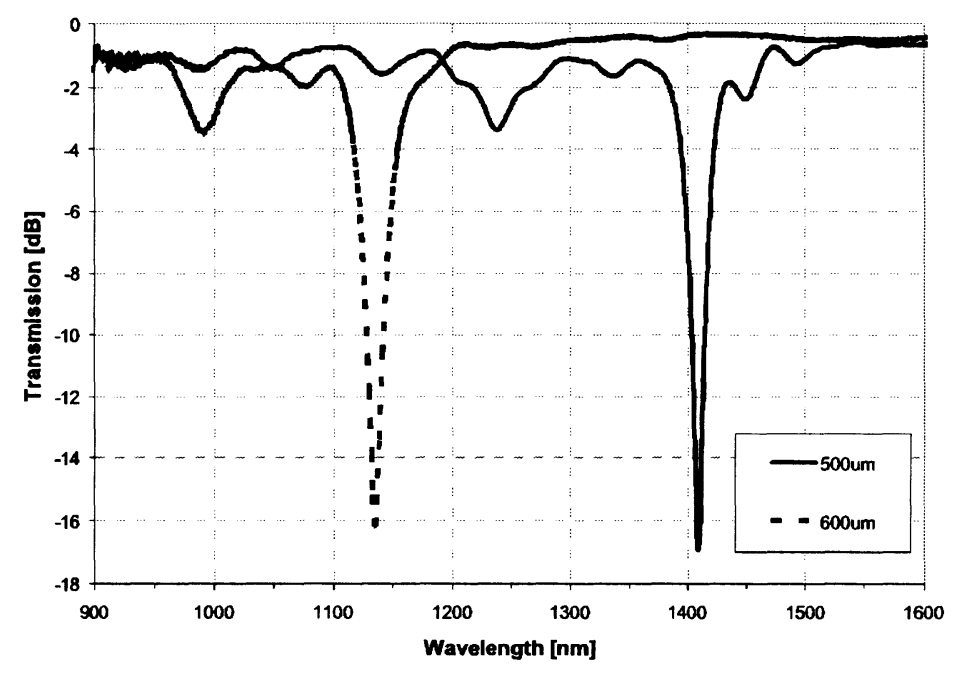

Fig.1. Transmission spectra for different grating periods

Recently developed photonic crystal fiber (PCF) offer great potential in designing LPGs since the waveguiding properties can be controlled by periodic adjustments of the airhole geometry as opposed to periodic variations of refractive index of the fiber core. The major drawback of the latter approach is a need of using photosensitive materials to inscribe gratings which makes them deteriorate. Previously, LPGs have been produced in nonphotosensitive PCFs by means of collapsing the holes caused by heat treatment with a $\mathrm{CO}_{2}$ laser [1], using an electric arc [2, 3], and application of mechanical stress [4]. LPGs inscribed by electric arc in PCF are capable of sensing strain and bending and are insensitive to temperature [3]. In this paper we present a comprehensive eigenmode analysis of the guided modes supported by the typical PCF and establish a modified phase matching conditions. The results are compared to the measured transmission spectra for LPGs with periods of 500 $\mu \mathrm{m}$ and 
$600 \mu \mathrm{m}$ shown in Fig 1. LPGs were fabricated by application of electric arc using a procedure described in [2] and [3]. We have compared generated dispersion map with the measured transmission spectra and detected resonant modes for each attenuation band within the given wavelength range. All the signature features of the observed transmission spectra are explained by multiple-period resonances between cladding modes supported by bulky silica cladding and a core mode.

\section{Modal analysis of PCF}

We studied LPGs inscribed in the endlessly singlemode PCF supplied by Blaze Photonics. This PCF is all pure silica. The diameter of the core was $12 \mu \mathrm{m}$ and it was surrounded by 54 airholes, with the space between adjacent holes being $8 \mu \mathrm{m}$. Air holes are arranged in a shape of hexagonal pattern with one missing hole in the middle. A missing hole forms a fibre core and this particular PCF has a relatively thick bulk cladding separated from central core by just a few rings of air holes.

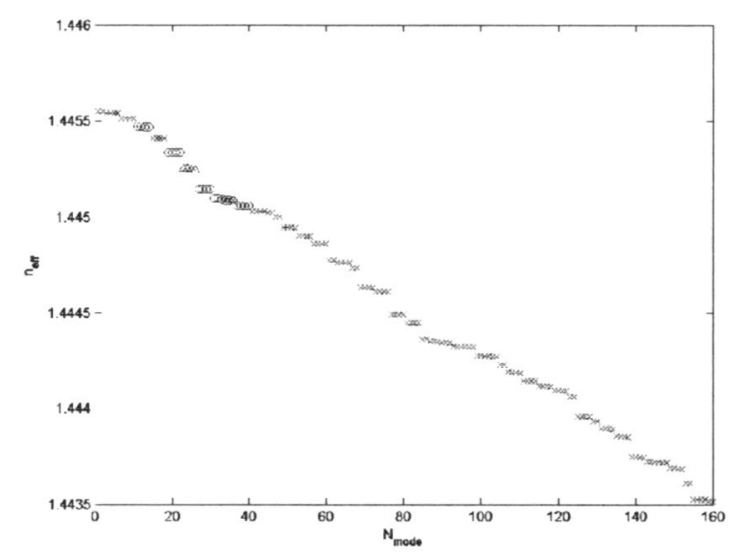

a

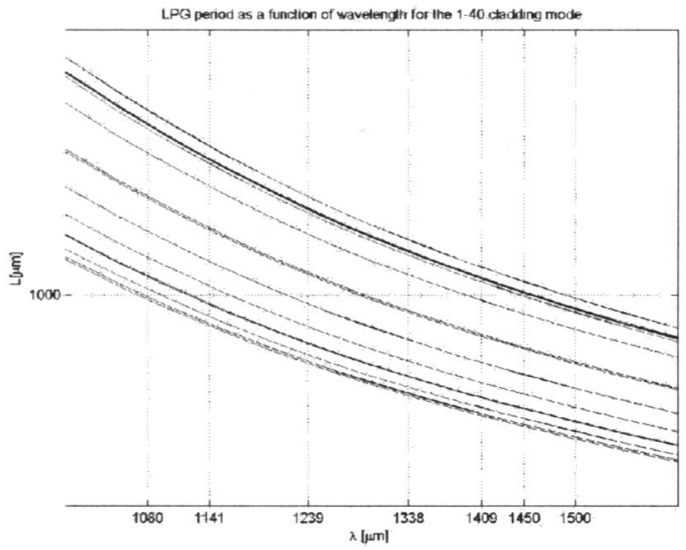

$\mathrm{b}$

Fig 2. Guided modes supported by PCF: a) Effective refractive index versus mode order

b) Dispersion of LPG period required for phase matching $\Lambda_{\mathrm{eff}}=2 \pi\left|\beta_{\mathrm{co}}-\beta_{\mathrm{cl}}\right|$ for the lowest 40 modes

Guided modes and corresponding propagation constants and effective refractive indices were found by means of full-vectorial Maxwell finite element solver from commercial package FEMLAB [5]. Eigenmodes were two-, four-, or six-fold degenerated corresponding to different polarisations and six-fold symmetry of the problem. This geometry supports a large number of cladding modes similar to those of ring resonator formed by the bulky cladding ring. It turns out that the mode localised in the core in not a fundamental mode for such a waveguide and its order is $148^{\text {th }}$. The lowest order modes are actually cladding modes as bulky cladding allows for more spacious modes. There is a naturally expected correspondence between the mode order and mode special scale - the higher the order the finer is special structure of the mode. When the special scale of the mode compares to the core diameter it corresponds to a core mode. Effective refractive index decreases with the mode number so that effective indices of many cladding modes exceed that of the core mode as shown in Fig 2a. Such behaviour stems from the fact that cladding modes propagate through the pure silica cladding and there interaction with the holey cladding is weaker compared to the core mode. Physically speaking, this is similar to the situation for all-silica W-shaped fibre with depressed cladding. We have confirmed this similarity by direct comparison of modes of Blaze PCF and corresponding propagation constants with a W-shaped fibre in which holey area is replaced with a homogeneous material of refractive index equal to the geometrically averaged refractive index of the air holes and silica.

\section{Multiple Period Resonances}

In order to identify modes responsible for resonant coupling to a core mode, a conventional phase matching condition $L=2 \pi\left|\beta_{c o}-\beta_{c l}\right|$, where $L$ is a beating period, $\beta_{c o}$ and $\beta_{c l}$ are the propagation constants of core and cladding modes. Typically, one LPG period $\Lambda=L$ is enough to accumulate phase difference $2 \pi$ required for phase matching. However, in the considered case of PCF a single grating period is not enough to provide phase matching with any of cladding modes for wavelength up to $2.2 \mu \mathrm{m}$. Phase matching can only be achieved after longer propagation e.g. a beating length should be a multiple of grating period. The results of phase matching analysis are presented in Fig $2 \mathrm{~b}$. It shows a beating period $L=2 \pi\left|\beta_{c o}-\beta_{c \mid}\right|$ as a function of wavelength and horizontal gridlines correspond to double 
period for the two gratings with periods of 500 and 600 microns. These results confirm the observation that the gratings with longer period have blue shifted resonances [3, 4].

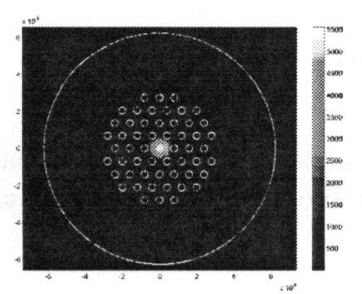

a

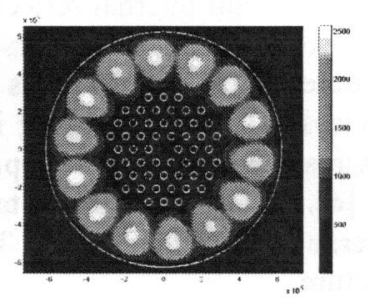

$\mathrm{d}$

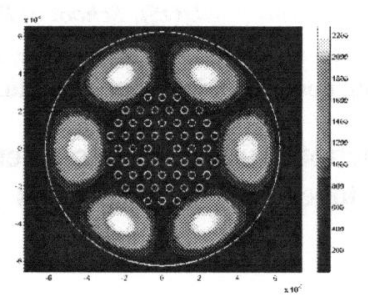

b

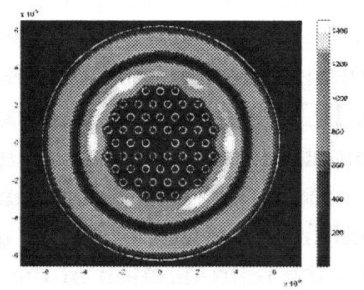

$\mathrm{e}$

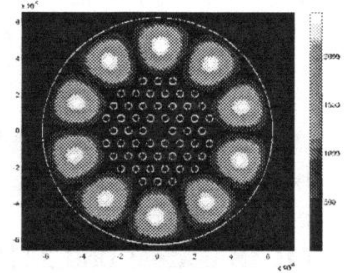

c

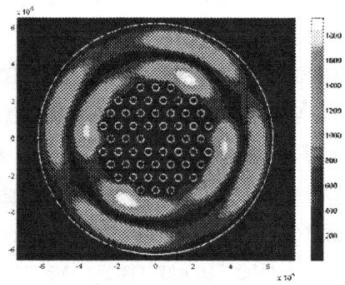

f

Fig 3. Resonant modes corresponding to double period resonances for LPG wiz $\Lambda=500 \mu \mathrm{m}$ : a) core mode at $\lambda=1400 \mathrm{~nm}$ b) $11^{\text {th }}$ mode at $\lambda=1141 \mathrm{~nm} \mathrm{c)} 20^{\text {th }}$ mode at $\lambda=1239 \mathrm{~nm}$ d) $27^{\text {th }}$ mode at $\lambda=1409 \mathrm{~nm}$ e) $31^{\text {st }}$ mode at $\lambda=1450 \mathrm{~nm}$ d) $27^{\text {th }}$ mode at $\lambda=1492 \mathrm{~nm}$

Figure 3 shows the intensity profiles of the modes corresponding to double period resonances for LPG with period of $500 \mu \mathrm{m}$. Another argument to support the suggested explanation of the observed transmission spectra is the strain sensitivity of the main resonance at $1409 \mathrm{~nm}$ for LPG with period $500 \mu \mathrm{m}$ which was measured to be $\delta \lambda / \delta \sigma=2.04 \pm 0.12 \mathrm{pm} / \mu \varepsilon$ where $\sigma$ is strain [3]. Linear regression of the dispersion curve for 27th-30th resonant mode around $1409 \mathrm{~nm}$ gives the sensitivity $\delta \lambda / \delta \sigma=2.14 \mathrm{pm} / \mu \varepsilon$.

\section{Conclusion}

Numerical modal analysis of the photonic crystal fiber fibre with thick bulk cladding is performed to analyse phase matching conditions between core- and cladding modes propagating along long period gratings. All the signature features of the experimentally observed transmission spectra are explained by multiple period resonances between these modes.

\section{References}

1. G. Kakarantzas, T. A. Birks, and P. St. J. Russell, “Structural long-period gratings in photonic crystal fibers,” Opt. Lett. 27, 1013-1015 (2002)

2. A. Malki, G. Humbert, Y. Ouerdane, A. Boukhenter, and A. Boudrioua, "Investigation of the writing mechanism of electric arc induced long-period fiber gratings," Appl. Opt. 42, 3776-3779 (2003)

3. H. Dobb, K. Kalli and D. J. Webb, "Temperature-insensitive long period grating sensors in photonic crystal fibre," Electron. Lett. 40, (2004)

4. J. H. Lim, K. S. Lee, J. C. Kim, and B. H. Lee, "Tunable fiber gratings fabricated in photonic crystal fiber by use of mechanical pressure," Opt. Lett. 29, 331-333 (2004)

5. www.comsol.com 